\title{
Breakthrough Fusarium solani fungaemia in a patient with acute myeloid leukaemia receiving posaconazole prophylaxis after three days concomitant omeprazole
}

\author{
Karina O’ Connell, John Kelehan, Martin Cormican \\ Michael A Petrou, Amjad Hayat, Deirbhile Keady
}

\begin{abstract}
Introduction: Disseminated Fusarium infection carries a very high mortality therefore prevention remains the cornerstone in management of at risk patients. This approach incorporates the use of an appropriate broad spectrum antifungal agent with activity against both yeast and filamentous fungi. We report a case of breakthrough Fusarium solani infection in a 66-year-old neutropaenic female patient with acute myeloid leukaemia while on posaconazole prophylaxis. Case report: The patient was admitted with partial opacification of her maxillary sinuses and we believe that this served as the likely focus of infection. The fungus was isolated from blood 17 days post posaconazole prophylaxis and three days post omeprazole treatment though the Minimum Inhibitory Concentration (MIC) to posaconazole using the Clinical and Laboratory Standards Institute (CLSI) recommended
\end{abstract}

Karina O' Connell', John Kelehan', Martin Cormican², Michael A Petrou ${ }^{3}$, Amjad Hayat ${ }^{4}$, Deirbhile Keady ${ }^{1}$

Affiliations: ${ }^{1}$ Department of Medical Microbiology, University College Hospital, Galway, Ireland; ${ }^{2}$ Department of Bacteriology, National University of Ireland, Galway, Ireland; 'Department of Mycology, Imperial Healthcare NHS Trust, London, UK; ${ }^{4}$ Department of Haematology, University College Hospital, Galway, Ireland.

Corresponding Author: Dr. Karina O' Connell, Department of Medical Microbiology, University College Hospital, Galway, Ireland; Phone: 35391544573; Email: karinaoconnell@gmail.com

Received: 03 February 2011

Accepted: 26 March 2011

Published: 31 May 2011 method was very low. Three weeks treatment with liposomal amphotericin $B$ proved to be successful, however, due to a rising creatinine the patient was subsequently changed to voriconazole with mycological and clinical cure. Conclusion: This case highlights the need for full histopathological and microbiological/ mycological investigations in patients with sinus opacification or mucosal thickening, alertness to Gram stain interpretation from blood cultures and above all, awareness that when proton pump inhibitors are used they might reduce triazole absorption.

Keywords: Breakthrough, Fusarium, Posaconazole, Omeprazole, Fungaemia, Sinusitis

$* * * * * * * * *$

Connell KO, Kelehan J, Cormican M, Petrou MA, Hayat A, Keady D. Breakthrough Fusarium solani fungaemia in a patient with acute myeloid leukaemia receiving posaconazole prophylaxis after three days concomitant omeprazole. International Journal of Case Reports and Images 2011;2(5):7-12.

$* * * * * * * * *$

doi:10.5348/ijcri-2011-05-32-CR-2

\section{INTRODUCTION}

Posaconazole is a triazole antifungal with a broad spectrum of activity against both yeasts and filamentous fungi and is indicated for prophylaxis of invasive fungal infection in patients receiving remission-induction chemotherapy for acute myeloid leukemia expected to result in prolonged neutropenia and who are at high risk of developing invasive fungal 


\section{infections. [1]}

Breakthrough fungal infections during therapy or prophylaxis are not uncommon and the main reasons could be intrinsic resistance of the fungus, acquired resistance during therapy, low or lack of activity of the antifungal (narrow spectrum agent), inadequate levels of drug due to low dosages or erratic absorption and no circulating drug as in the case of patients receiving topical polyene antifungal prophylaxis. $[2,3]$ Due to increased risk of gastrointestinal hemorrhage in many of these patients resulting from a combination of steroid treatment and thrombocytopenia, many patients receive gastric acid suppressants. There is one reported case in the literature of omeprazole significantly reducing posaconazole serum trough levels with no perceived clinical impact. We now present a case of fusariosis potentially resulting from such an interaction.

\section{CASE REPORT}

A 66-year-old female was referred to the hematology services in January 2009 with symptoms of sinusitis and lethargy ongoing for one month. Bloods on admission revealed a haemoglobin level of $5.4 \mathrm{~g} / \mathrm{dl}$ and a white cell count of $117.5 \times 10^{9} / \mathrm{L}$ comprising blasts $92.83 \times 10^{9} / \mathrm{L}$, neutrophils $16.5 \times$ $10^{9} / \mathrm{L}$, lymphocytes $5.9 \times 10^{9} / \mathrm{L}$, monocytes $1.2 \times 10^{9} / \mathrm{L}$ and metamyelocytes $1.2 \times 10^{9} / \mathrm{L}$. Platelets were within normal range. A chest $\mathrm{x}$-ray was carried out and was unremarkable. An x-ray of sinuses revealed partial opacification of the right maxillary and ethmoidal sinuses but no pathological investigations were undertaken (Figure 1). The patient had not received any treatment prior to referral to our institute. A bone marrow aspirate was performed and a diagnosis of acute myeloid leukemia without maturation and positive for the FLT3 mutation was made. A tunneled central venous catheter was placed to facilitate administration of chemotherapy and treatment commenced with idarubicin/cytarabine. Antifungal and antiviral prophylaxis was commenced simultaneously with posaconazole $200 \mathrm{mg}$ tds po and valaciclovir $500 \mathrm{mg}$ o.d. p.o. respectively.

On day two of her chemotherapy she became profoundly neutropenic with a white cell count of $1 \mathrm{x}$ $10^{9} / \mathrm{L}$. This was expected as a result of therapy. She developed a fever of $38.3^{\circ} \mathrm{C}$ and a full septic screen was carried out, as there was no obvious focus of infection on examination. She was commenced on empirical antibiotic treatment with piperacillin/ tazobactam 4.5 g t.d.s. intravenously (i.v.). Over the subsequent two weeks she continued to experience multiple temperature spikes $>39^{\circ} \mathrm{C}$. During this time she had further imaging of her sinuses and a CT scan showed extensive mucosal thickening of the right maxillary sinus and some mild mucosal thickening of the base of the left maxillary sinus (Figure 2). Once more, no surgical or further investigations were carried out. Her antimicrobial treatment had been broadened out in the

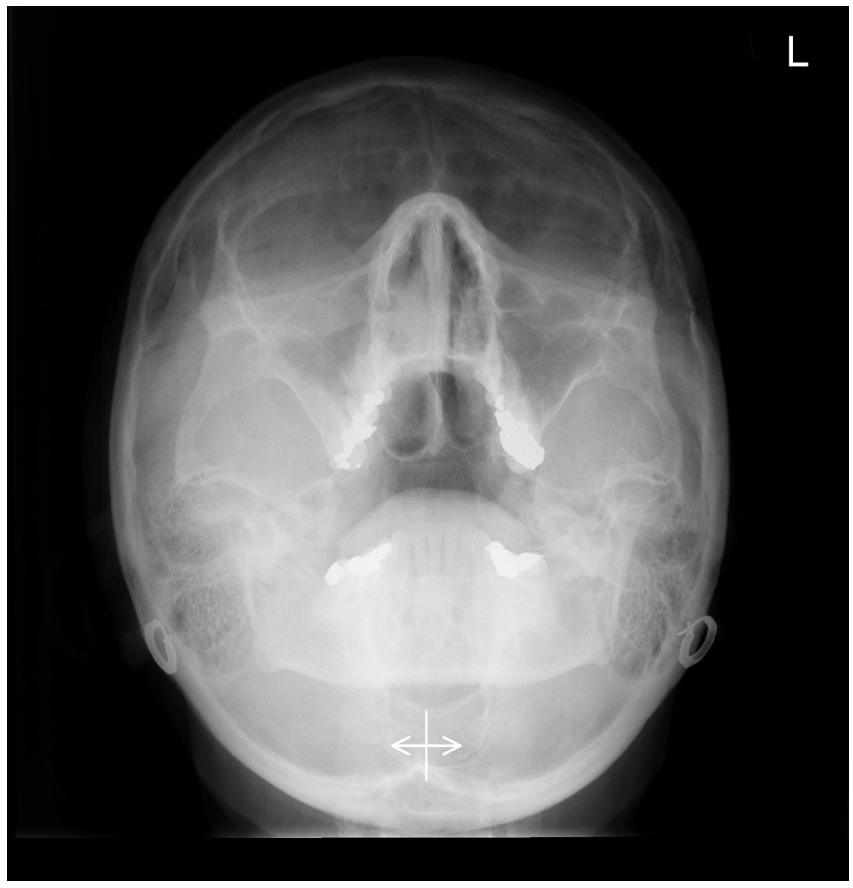

Figure 1: An x-ray of sinuses revealed partial opacification of the right maxillary and ethmoidal sinuses.

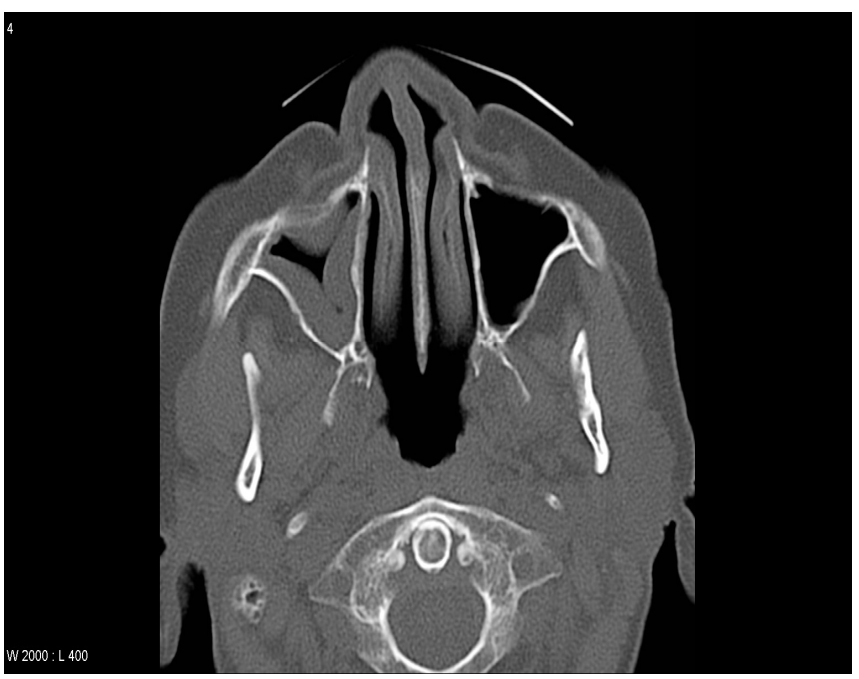

Figure 2: CT scan showed extensive mucosal thickening of the right maxillary sinus and some mild mucosal thickening of the base of the left maxillary sinus.

interim to include vancomycin $15 \mathrm{mg} / \mathrm{kg}$ bd iv and ciprofloxacin $500 \mathrm{mg}$ b.d. orally (p.o.). On day 15, omeprazole $20 \mathrm{mg}$ o.d. p.o., a gastric acid suppressant was added to her medications as a precautionary measure as her platelet count had dropped to $<20 \mathrm{x}$ $10 \% / \mathrm{L}$. Of note, the patient did not have any gastrointestinal disturbance that could have potentially interfered with the absorption of posaconazole. On day 20, liposomal amphotericin B $5 \mathrm{mg} / \mathrm{kg}$ iv was commenced and the patient's hickman line was 
removed. On the same day, the blood cultures drawn from both the hickman line and peripherally on day 18 became positive.

Gram-stain revealed darkly staining (gram positive) yeast like cells. The culture, which was a presumptive yeast, was subcultured to chromogenic Candida agar (Oxoid Brilliance ${ }^{\mathrm{TM}}$ Candida agar) and Sabourauds dextrose agar. At 24 hours incubation the morphology of the colonies was atypical for Candida spp. and the organism failed to identify using the VITEK 2. A lactophenol cotton blue preparation showed multiple hyaline septate hyphae and macroconidia, typical of Fusarium spp. (Figure 3). After five days incubation, colonies with typical morphology for Fusarium spp. (flat woolly colonies, lavender in colour) were seen on the Sabourauds dextrose agar plate, which was confirmed as $F$. solani. Antifungal susceptibility was performed using the CLSI recommended method and the minimum inhibitory concentrations were: for amphotericin B: $0.125 \mu \mathrm{g} / \mathrm{ml}$, fluconazole: $>64 \mu \mathrm{g} / \mathrm{ml}$, posaconazole: $0.125 \mu \mathrm{g} / \mathrm{ml}$, voriconazole: $0.125 \mu \mathrm{g} / \mathrm{ml}$, itraconazole: $0.125 \mu \mathrm{g} / \mathrm{ml}$, caspofungin: $>8 \mu \mathrm{g} / \mathrm{ml}$, micafungin: $>8 \mu \mathrm{g} / \mathrm{ml}$, and flucytosine $>32 \mu \mathrm{g} / \mathrm{ml}$. Posaconazole was discontinued on day 24.

Sterile blood cultures were obtained four days into treatment with liposomal amphotericin B. An ophthalmology review was sought and fundoscopy did not demonstrate any intraocular fungal infection. Seven days into treatment with liposomal amphotericin B, a number of erythematous cutaneous nodules and plaques were observed on the patient's lower limbs. The appearance was consistent with cutaneous manifestation of Fusarium infection (Figure 4). Two $4 \mathrm{~mm}$ punch biopsies were taken and submitted for fungal culture and histology. There was no growth on Sabourauds dextrose agar after eight days incubation. The histological appearances of the biopsy were non-specific and specialised fungal stains did not reveal any fungi.

On day 40, after 20 days of treatment, amphotericin B was discontinued due to creatinine

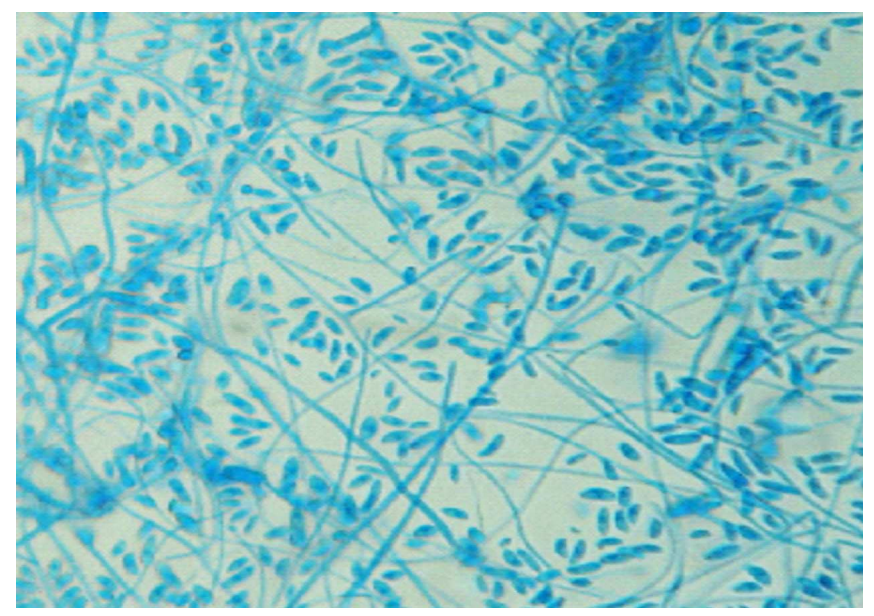

Figure 3: Lactophenol cotton blue preparation.
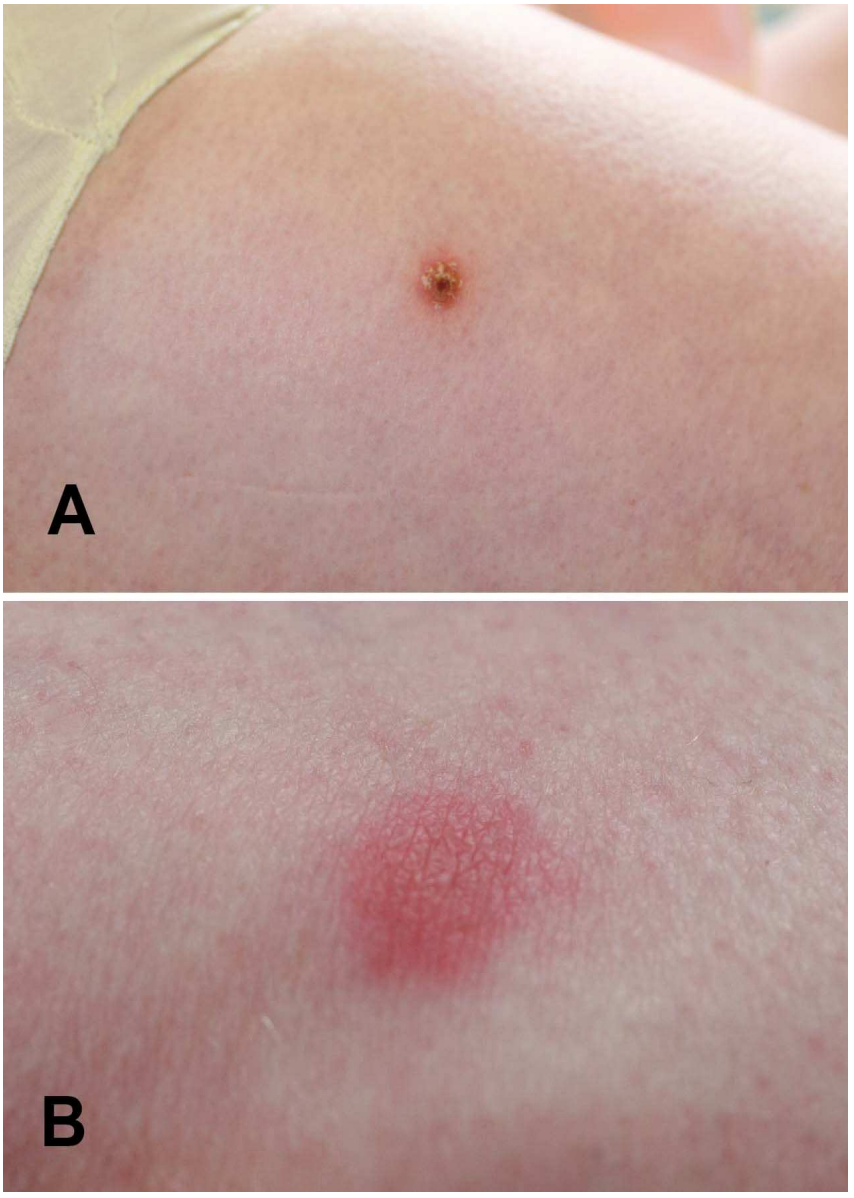

Figure 4: A) Plaque right thigh, B) Erythematous nodule left lower limb.

elevation to $213 \mu \mathrm{mol} / \mathrm{L}$ and treatment was continued with voriconazole $200 \mathrm{mg}$ b.d. iv. The patient's neutrophil count later recovered and the skin lesions and sinus symptoms resolved completely thus achieving both clinical and mycological cure. She was discharged home on oral voriconazole 200mg BD with a plan to continue treatment throughout her chemotherapy regime. There was no recurrence of Fusarium infection; however, the patient died 6 weeks later from complications relating to her underlying haematological malignancy.

\section{DISCUSSION}

After Aspergillus spp., Fusarium spp. is the second most frequent cause of invasive filamentous fungal infection in immunocompromised patients. Disseminated Fusarium infection carries a very high mortality even with aggressive antifungal therapy therefore prevention of infection is the cornerstone in managing at risk patients. This involves a multifactorial approach incorporating minimization of exposure to the fungus in high-risk patients and 
increasing the exposure to the antifungal drug chosen for prophylaxis $[2,4]$.

Fusarium breakthrough has been seen during treatment with fluconazole or caspofungin and during voriconazole prophylaxis $[5,6,7]$. It is not surprising that Fusarium breakthrough occurred during fluconazole and during caspofungin treatment as neither of the two drugs has in vivo or in vitro activity, whereas breakthrough during voriconazole treatment or prophylaxis requires full investigation to find out whether this was a result of inadequate drug levels or acquired resistance, as voriconazole is a highly active drug against Fusarium spp. Though there are several reports of breakthrough Zygomycosis and other fungi in patients receiving posaconazole prophylaxis [8-12] and two case reports of breakthrough Fusarium spp. in patients receiving voriconazole prophylaxis, [7, 13] there are no previous reports of breakthrough Fusarium infection in a patient on posaconazole prophylaxis.

It is evident that our patient was admitted with probable fusarial sinusitis as both the X-ray and CT scan findings were consistent with invasive fungal infection. It is unfortunate that samples for microbiological/ mycological investigations were not taken once the opacification was noticed on admission and when mucosal thickening was reported on CT scan during the two weeks of intermittent fever despite broad spectrum antibacterial therapy. The skin lesions appearing on treatment were clinically consistent with cutaneous Fusarium infection and this was considered to be the most likely cause given the high percentage $(>50 \%)$ of immunocompromised patients who exhibit skin lesions [14]. The histological specimens were inconclusive and perhaps this is due to the antifungal treatment that the patient had received before the biopsies were taken.

The bloodstream infection in our patient was with an apparently susceptible strain of $F$. solani presenting as a febrile episode in a profoundly neutropenic patient following 17 days of posaconazole prophylaxis. The case illustrates the continuing need for a high index of suspicion for fungal infection in these patients even whilst receiving appropriate antifungal prophylaxis. Our case also indicates that when abnormalities are seen in the sinuses, either with X-rays or more importantly CT scan, a thorough investigation of invasive fungal infection including biopsies and or washouts for both microbiology/mycology and histopathology must be obtained. There is some basis in the literature for concern that posaconazole blood levels may be inadequate in a high proportion of patients on prophylactic doses and that this may be a greater risk in those with gastrointestinal disturbance (not applicable in this case) and those taking drugs to reduce gastric acidity such as cimetidine and proton pump inhibitors $[15,16]$.

The breakthrough infection in our patient occurred although prophylaxis was initiated promptly on commencement of chemotherapy and the subsequent isolate had a low MIC to posaconazole. The patient though febrile during the first two weeks following admission remained free of systemic infection and the blood cultures only became positive with what looked like yeast on the third day of omeprazole treatment. The patient did not have any gastrointestinal disturbance and omeprazole was added as a precaution and this ought to have been the reason that permitted the otherwise contained infection to disseminate. Although there are no defined clinical breakpoints for Fusarium and posaconazole, the MIC of $0.125 \mu \mathrm{g} / \mathrm{mL}$ is below achievable concentrations of posaconazole using recommended dosing regimens and thus it is expected to suppress fungal growth.

The main organisms isolated from blood are bacteria and yeasts. Filamentous fungi are very rarely isolated from blood and when they are, the first step in the identification process, the gram stain may yield misleading information. One might assume in the absence of an expert mycologist, that the organism isolated is a yeast. There is also the added danger of calling all yeasts Candida spp. or Candida nonalbicans when they are germ tube negative, thus tempting the clinicians into using an echinochandin to treat what it is ultimately an echinochandin resistant filamentous fungus. Fusarium spp., like Aspergillus terreus are the commonest filamentous fungi found in blood cultures as both sporulate in vivo [17].

In retrospect, given the clinical presentation, radiological findings and clinical course, it is very likely that localised infection in the maxillary sinus was present at the time of initiation of chemotherapy. If this was the case, this may have contributed to the establishment of blood stream infection whilst on posaconazole prophylaxis. More significant for the role of posaconazole in prophylaxis of fungal infection is the possibility that a drug interaction with omeprazole, a proton pump inhibitor (PPI) may have impacted on levels of posaconazole and reduced its efficacy. We did not however measure posaconazole levels and therefore were unable to demonstrate a decline in the posaconazole level following introduction of omeprazole.

Cimetidine, an H2-receptor antagonist reduces the bioavailability of posaconazole and the manufacturers advise that the co-administration of cimetidine and posaconazole should be avoided if possible [18]. Studies carried out by Courtney et al. (2004) suggest that posaconazole absorption is not $\mathrm{pH}$ dependent, however, the manufacturers advise that coadministration of posaconazole with PPIs should also be avoided if possible $[19,20]$ The potential for an interaction between PPIs and posaconazole has previously been proposed and a case report from the Netherlands in 2009 describes a 58-year-old male patient who was treated for invasive aspergillosis and experienced a significant decrease in the posaconazole serum trough level post introduction of omeprazole 40 mg once daily $[16,21]$. Omeprazole was administered for three days and the authors noted that posaconazole serum levels increased again to baseline on discontinuation of omeprazole [16]. 
More recently, Lebeaux et al. carried out a study assessing the prevalence of low plasma posaconazole concentrations in patients on both prophylaxis and curative treatment [15]. The authors reported low posaconazole levels in $44 \%(16 / 36)$ of patients in the prophylaxis group. Low levels were more frequent amongst patients with diarrhoea or mucositis. Two patients in the prophylaxis group with low posaconazole levels experienced possible invasive fungal infection [15]. Based on their findings they recommend therapeutic drug monitoring of posaconazole for immunosuppressed patients with gastrointestinal disorders; we support their recommendations but we must add that this test is not easily accessible to the majority of institutions.

\section{CONCLUSION}

We conclude therefore, that the patient in this case had an undiagnosed maxillary sinusitis caused by $F$. solani, prior to becoming neutropenic. The infection was most likely being suppressed by posaconazole at prophylactic doses. The patient then experienced a breakthrough invasive infection three days following co-administration of omeprazole with posaconazole, potentially due to altered absorption of posaconazole. This case highlights the need for therapeutic drug monitoring in patients in receipt of posaconazole prophylaxis who are immunosuppressed and in whom gastric acid suppressants are required.

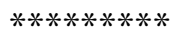

\section{Acknowledgements}

Dr. Triona Walshe of University College Hospital, Galway for providing the CT images.

\section{Author Contributions}

Karina O' Connell - Substantial contributions to conception and design, Acquisition of data, Analysis and interpretation of data, Drafting the article, Revising it critically for important intellectual content, Final approval of the version to be published

John Kelehan - Substantial contributions to conception and design, Acquisition of data, Analysis and interpretation of data, Drafting the article, Revising it critically for important intellectual content, Final approval of the version to be published

Martin Cormican - Substantial contributions to conception and design, Acquisition of data, Analysis and interpretation of data, Drafting the article, Revising it critically for important intellectual content, Final approval of the version to be published

Michael A Petrou - Substantial contributions to conception and design, Acquisition of data, Analysis and interpretation of data, Drafting the article, Revising it critically for important intellectual content, Final approval of the version to be published Amjad Hayat - Substantial contributions to conception and design, Acquisition of data, Analysis and interpretation of data, Drafting the article, Revising it critically for important intellectual content, Final approval of the version to be published

Deirbhile Keady - Substantial contributions to conception and design, Acquisition of data, Analysis and interpretation of data, Drafting the article, Revising it critically for important intellectual content, Final approval of the version to be published

\section{Guarantor}

The corresponding author is the guarantor of submission.

\section{Conflict of Interest}

Authors declare no conflict of interest.

\section{Copyright}

(C) Karina O' Connell et al. 2011; This article is distributed under the terms of Creative Commons attribution 3.0 License which permits unrestricted use, distribution and reproduction in any means provided the original authors and original publisher are properly credited. (Please see www.ijcasereportsandimages.com /copyright-policy.php for more information.)

\section{REFERENCES}

1. Cornely OA, Böhme A, Buchheidt D, Einsele H, Heinz WJ, Karthaus M, Krause SW, Krüger W, Maschmeyer G, Penack O, Ritter J, Ruhnke M, Sandherr M, Sieniawski M, Vehreschild JJ, Wolf $\mathrm{HH}$, Ullmann AJ. Primary prophylaxis of invasive fungal infections in patients with hematologic malignancies. Recommendations of the Infectious Diseases Working Party of the German Society for Haematology and Oncology. Haematologica 2009, 94:113-122.

2. Winston DJ, Bartoni K, Territo MC, Schiller GJ. Efficacy, Safety, and Breakthrough Infections Associated with Standard Long-Term Posaconazole Antifungal Prophylaxis in Allogeneic Stem-Cell Transplant Recipients. Biol Blood Marrow Transplant 2010: doi:10.1016/j.bbmt.2010.04.017.

3. Vehreschild JJ, Rüping MJ, Wisplinghoff $H$, Farowski F, Steinbach A, Sims R, Stollorz A, Kreuzer KA, Hallek M, Bangard C, Cornely OA. Clinical effectiveness of posaconazole prophylaxis in patients with acute myelogenous leukaemia (AML): a 6 year experience of the Cologne AML cohort. J Antimicrob Chemother 2010;65:1466-1471.

4. Nucci M, Anaissie E. Fusarium infections in immunocompromised patients. Clin Microbiol Rev 2007; 20:695-704.

5. Tascini C, Ferranti S, Leonildi A, Menichetti F. Breakthrough Fusarium sp probable pneumonia during fluconazole therapy in an AIDS patient with diabetes, candidemia, Pneumocystis carinii pneumonia and cytomegalovirus disseminated infection. J Chemother 2006;18:227-228.

6. Tascini C, Urbani L, Doria R, Catalano G, Leonildi 
A, Filipponi F, Menichetti F. Breakthrough Fusarium spp fungemia during caspofungin therapy in an $\mathrm{ABO}$-incompatible orthotopic liver transplant patient. J Chemother 2009;21:236-238.

7. Cudillo L, Girmenia C, Santilli S, Picardi A, Dentamaro T, Tendas A, de Fabritiis P. Breakthrough fusariosis in a patient with acute lymphoblastic leukemia receiving voriconazole prophylaxis. Clin Infect Dis 2005;40:1212-1213.

8. Kishel JJ, Sivik J. Breakthrough invasive fungal infection in an immunocompromised host while on posaconazole prophylaxis: an omission in patient counselling and follow-up. J Oncol Pharm Pract 2008;14:189-193.

9. Schlemmer F. Lagrange-Xélot M, Lacroix C, de la Tour R, Socié G, Molina JM. Breakthrough Rhizopus infection on posaconazole prophylaxis following allogeneic stem cell transplantation. Bone Marrow Transplant 2008;42:551-552.

10. Ananda-Rajah MR, Grigg A, Slavin MA. Breakthrough disseminated Scedosporium prolificans infection in a patient with relapsed leukaemia on prolonged voriconazole followed by posaconazole prophylaxis. Mycopathologica 2008; 166:83-86.

11. Lekakis LJ, Lawson A, Prante J, Ribes J, Davis GJ, Monohan G, Baraboutis IG, Skoutelis AT, Howard DS. Fatal rhizopus pneumonia in allogeneic stem cell transplant patients despite posaconazole prophylaxis: two cases and review of the literature. Biol Blood Marrow Transplant 2009; 15:991-5.

12. Mousset S, Bug G, Heinz WJ, Tintelnot K, Rickerts V. Breakthrough zygomycosis on posaconazole prophylaxis after allogeneic stem cell transplantation. Transpl Infect Dis 2010; 12:261-4.

13. Tomas JF, Delgado P, Garcia-Zueco JC, de Ona R, Estevez M, Rezusta A, Yague D, Garcia-Cia JI, Iglesias R, Rubio-Felix D. Safety and efficacy of primary prophylaxis with voriconazole in adult patients with acute leukaemia. Blood $2007\left(49^{\text {th }}\right.$ Ann Mtg Am Soc Hematol). Abstr no:4373.

14. Nucci M, Anaissie E. Cutaneous infection by Fusarium species in healthy and immunocompromised hosts: implications for diagnosis and management. Clin Infect Dis 2002; 35:909-20.

15. Lebeaux D, Lanternier F, Elie C, Suarez F, Buzyn A, Viard JP, Bougnoux ME, Lecuit M, Jullien V, Lortholary O. Therapeutic drug monitoring of posaconazole: a monocentric study with 54 adults. Antimicrob Agents Chemother 2009; 53:5224-9.

16. Alffenaar JW, van Assen S, van der Werf TS, Kosterink JG, Uges DR. Omeprazole significantly reduces posaconazole serum trough level. Clin Infect Dis 2009; 48:839.

17. Christie JD. Diagnosis of invasive mold infection. Is PCR the answer? Am J Clin Pathol 2003; 119:11-3.

18. Kosoglou T, Perentesis GP, Affrime MB, et al. The effect of antacid and cimetidine on the oral absorption of the antifungal agent $\mathrm{SCH}$ 39304. J Clin Pharmacol 1990; 30:638-42.

19. Courtney R, Radwanski E, Lim J, Laughlin M. Pharmacokinetics of posaconazole coadministered with antacid in fasting or nonfasting healthy men. Antimicrob Agents Chemother 2004; 48:804-8.

20. Noxafil (posaconazole) UK summary of product characteristics. Schering-Plough Ltd. February 2, 2006. 